\title{
A Mobile Tryptophan is the Intrinsic Charge Transfer Donor in a Flavoenzyme Essential for Nikkomycin Antibiotic Biosynthesis ${ }^{\dagger}$
}

\author{
Robert C. Bruckner, Gouhua Zhao, Patricia Ferreira, and Marilyn Schuman Jorns ${ }^{\star}$ \\ Department of Biochemistry and Molecular Biology, Drexel University College of Medicine, \\ Philadelphia, PA 19102
}

\section{Abstract}

The flavoenzyme nikD is required for the biosynthesis of nikkomycin antibiotics. NikD exhibits an unusual long wavelength absorption band attributed to a charge transfer complex of FAD with an unknown charge transfer donor. NikD crystals contain an endogenous active site ligand. At least four different compounds are detected in nikD extracts, including variable amounts of two ADP derivatives that bind to the enzyme's dinucleotide binding motif in competition with FAD, picolinate $(0.07 \mathrm{~mol} / \mathrm{mol} \mathrm{nikD})$ and an unknown picolinate-like compound. Picolinate, the product of the physiological catalytic reaction, matches the properties deduced for the active site ligand in nikD crystals. The charge transfer band is eliminated upon mixing nikD with excess picolinate but not by a reversible unfolding procedure that removes the picolinate-like compound, ruling out both compounds as the intrinsic charge transfer donor. Mutation of Trp355 to Phe eliminates the charge transfer band, accompanied by a 30 -fold decrease in substrate binding affinity. The results provide definitive evidence for Trp355 as the intrinsic charge transfer donor. The indole ring of Trp355 is coplanar with or perpendicular to the flavin ring in "open" or "closed" crystalline forms of nikD, respectively. Importantly, a coplanar configuration is required for charge transfer interaction. Absorption in the long wavelength region therefore constitutes a valuable probe to monitor conformational changes in solution that are likely to be important in nikD catalysis.

Nikkomycins are peptidyl nucleoside antibiotics that block the biosynthesis of chitin by inhibiting chitin synthase (1). Chitin, the second most abundant polysaccharide in nature, maintains the structural integrity of the cell wall in fungi and the exoskeleton of insects and other invertebrates. Neither chitin nor chitin synthase is found in mammals. Nikkomycins are effective for the therapeutic treatment of fungal infections in humans and as easily degraded insecticides in agriculture (2).

Biosynthesis of the nikkomycin peptide occurs via a nonribosomal pathway. The first step is catalyzed by an aminotransferase that converts L-lysine to an $\alpha$-keto intermediate that cyclizes and dehydrates to yield piperideine-2-carboxylate (P2C), a compound that can exist in imine and enamine forms (3). The second step is catalyzed by nikD in a reaction that involves a remarkable 4-electron oxidation of $\mathrm{P} 2 \mathrm{C}$ to picolinate, accompanied by reduction of $2 \mathrm{~mol}$ of oxygen to hydrogen peroxide (Scheme 1) (4-6). NikD contains 1 mol of covalently bound FAD (8"-S-cysteinyl-FAD), exists as a monomer in solution and acts as an obligate 2-electron acceptor. The initial 2-electron oxidation of P2C to dihydropicolinate (DHP) is rate-

\footnotetext{
${ }^{\dagger}$ This work was supported in part by Grant AI 55590 (M. S. J.) from the National Institutes of Health.

*To whom correspondence should be addressed. Phone: (215) 762-7495 FAX: (215) 762-4452., E-mail: E-mail: marilyn.jorns@drexelmed.edu.

${ }^{1}$ Abbreviations: FAD, flavin adenine dinucleotide; P2C, piperideine-2-carboxylate; DHP, dihydropicolinate; ES, electrospray ionization; Ade, adenine; FNR, ferredoxin-NADP ${ }^{+}$reductase.
} 
determining. Subsequent conversion of DHP to picolinate appears to proceed without release of the labile DHP intermediate into solution $(5,6)$.

NikD exhibits two absorption maxima in the visible region, a feature characteristic of flavincontaining enzymes. However, the enzyme also exhibits an atypical long wavelength absorption band, extending out to nearly $700 \mathrm{~nm}$. This absorption band can be eliminated by enzyme denaturation, flavin reduction, acidic $\mathrm{pH}\left(\mathrm{pK}_{\mathrm{a}}=7.3\right)$ or binding of competitive inhibitors. The long wavelength absorption band has been attributed to a charge transfer complex containing oxidized FAD as the acceptor and an unknown species as the charge transfer donor (5).

Crystal structures of open and closed forms of nikD have recently been solved at 1.9 and 1.15 $\AA$ resolution, respectively (7). Both forms contain an endogenous active site ligand that is modeled as an aromatic carboxylate with a 6-membered ring. The enzyme contains flavin- and substrate-binding domains. FAD is bound in an extended conformation with its ADP moiety bound to a classic dinucleotide binding motif in the flavin domain. The flavin ring is located at the interface between the flavin and substrate domains. Conversion from the open to the closed form involves a dramatic, large-scale rotation of the substrate domain and movement of a 5-residue loop into the active site cavity, accompanied by a striking rearrangement of key active site residues. For example, the aromatic ring of the endogenous ligand is stacked (3.5 $\AA$ ) above the flavin ring in the closed form and the indole ring of Trp355 is approximately perpendicular to the flavin ring. In contrast, the indole ring of Trp355 assumes a stacked position above the flavin ring in the open form, displacing the ligand in an impressive rearrangement that involves a 90 degree rotation of the aromatic rings of both the ligand and Trp355 (Figure 1).

Trp355 and the endogenous ligand emerged as likely candidates for the charge transfer donor, as judged by the observed crystal structures. In this paper, we identify the endogenous active site ligand and the intrinsic charge transfer donor. The results provide insight regarding the mechanism of substrate binding.

\section{EXPERIMENTAL PROCEDURES}

\section{Materials}

Picolinate, nicotinate, isonicotinate, benzoate, 2-pyrazine carboxylate, alkaline phosphatase, and purified phosphodiesterase I from Crotalus adamanteus venom were purchased from Sigma. 2-Pyrimidinecarboxylate and 4-pyrimidinecarboxylate were obtained from Bridge Organics. Restriction enzymes and T4 DNA ligase were purchased from New England Biolabs. Ni-NTA agarose ( $\mathrm{Ni}^{2+}$ affinity matrix) was obtained from Qiagen. P2C was prepared as previously described (6).

\section{Enzyme Purification and Assay}

Recombinant wild type nikD was isolated from cells (E. coli BL21(DE3)/pDV101) grown in LB or Terrific Broth, as previously described $(5,6)$. A clone that expressed the Trp355Phe mutant (E. coli BL21(DE3)/pGZ42) was constructed as described below and grown in LB. The mutant enzyme was isolated by the same method used for purification of wild type nikD. Enzyme activity was measured by monitoring picolinate formation at $264 \mathrm{~nm}$ in a standard assay containing $50 \mu \mathrm{M} \mathrm{P} 2 \mathrm{C}$ (6). Specific activity values are calculated based on flavin content and expressed as a turnover rate ( $\mu \mathrm{mol}$ picolinate $/ \mu \mathrm{mol} \mathrm{nikD} / \mathrm{min})$. Apparent steady state kinetic parameters were determined by measuring picolinate formation at various concentrations of $\mathrm{P} 2 \mathrm{C}$ in air-saturated $50 \mathrm{mM}$ potassium phosphate buffer, $\mathrm{pH} 8.0$, at $25^{\circ} \mathrm{C}$. 
The concentration of purified wild type or Trp355Phe mutant enzyme was determined based on its absorbance at $455\left(\varepsilon=11,200 \mathrm{M}^{-1} \mathrm{~cm}^{-1}\right)$ or $462 \mathrm{~nm}\left(\varepsilon=12,700 \mathrm{M}^{-1} \mathrm{~cm}^{-1}\right)$, respectively.

\section{Preparation of NikD Extracts}

NikD was heated at $100^{\circ} \mathrm{C}$ for $2 \mathrm{~min}$, centrifuged at $14,000 \times \mathrm{g}$ for $15 \mathrm{~min}$ and then ultrafiltered to remove denatured protein. Alternatively, the protein was denatured by addition of acetonitrile $(66 \%, \mathrm{v} / \mathrm{v})$ at $22{ }^{\circ} \mathrm{C}$. Denatured protein was removed by centrifugation after a 10 min incubation on ice. Prior to analysis, heat or acetonitrile extracts were concentrated under vacuum.

\section{Chromatography and Spectroscopy}

Unless otherwise indicated, HPLC analyses of nikD extracts were conducted at room temperature using a Rainin HPLC system equipped with a Hamilton PRP-X100 anion exchange column $(10 \mu \mathrm{m}$ particle, $4.6 \mathrm{~mm} \times 250 \mathrm{~mm})$ or a Varian Microsorb 100-5 C18 column $(5 \mu \mathrm{m}$ particle, $4.6 \mathrm{~mm} \times 250 \mathrm{~mm}$ ). The column eluate was monitored by its absorbance at $264 \mathrm{~nm}$. Anion exchange HPLC analyses were conducted by using isocratic elution at a flow rate of 1.4 $\mathrm{ml} / \mathrm{min}$ (method 1 ) or $1.2 \mathrm{~mL} / \mathrm{min}(\operatorname{method} 2)$. Method 1 corresponds to the previously described high resolution protocol for separation of positional isomers of picolinate (6). The elution solvent for method 2 was prepared by mixing (v/v) 1 part of $25 \mathrm{mM}$ ammonium carbonate $\mathrm{pH} 10.3$ with 1 part of 50\% methanol in water $(\mathrm{v} / \mathrm{v})$. Reversed phase HPLC analyses using method 3 were conducted at a flow rate of $1 \mathrm{~mL} / \mathrm{min}$ using a previously described elution protocol (8). Reversed phase HPLC analyses with method 4 were conducted using the following elution protocol (flow rate $=1 \mathrm{~mL} / \mathrm{min}$ ): $5 \mathrm{~min}$ isocratic elution with solvent $\mathrm{A}$ (100 $\mathrm{mM}$ ammonium acetate, $\mathrm{pH} 5.4) ; 25$ min linear gradient to 50\% solvent B (90\% methanol/ $10 \%$ water); 5 min linear gradient to $70 \%$ solvent B; 5 min linear gradient to $0 \%$ solvent B; 5 min isocratic elution with solvent A. Methods 1 and 3 were used for routine HPLC separations. Separations conducted in conjunction with mass spectral analyses employed methods 2 and 4 but different HPLC hardware, as indicated below.

As will be described, nikD extracts contain two ADP derivatives (compounds I and IV). Compound I is labile and decomposes to yield AMP under alkaline conditions. The amount of compounds I and IV in nikD extracts was determined by quantitative HPLC analysis using an AMP standard curve. Analyses were performed by using a reversed phase (compounds I, IV) and/or an anion exchange (compound I) column. Compound I in untreated ligand extracts was estimated based on the combined area under peaks due to the intact compound and its AMP decomposition product. In studies with extracts that had been incubated at alkaline $\mathrm{pH}$, compound I was estimated based on the area under the AMP peak.

Absorption spectra were recorded using an Agilent Technologies 8453 diode array or a Perkin Elmer lambda 25 spectrophotometer. To determine the stoichiometry of FAD incorporation into nikD, the enzyme was denatured with $3.0 \mathrm{M}$ guanidine hydrochloride. The concentration of FAD was estimated using an extinction coefficient previously determined for free FAD in 3.0 M guanidine hydrochloride $\left(\varepsilon_{450}=11,900 \mathrm{M}^{-1} \mathrm{~cm}^{-1}\right)(9)$. Protein concentration was determined based on the absorbance at $280 \mathrm{~nm}$ after enzyme denaturation using an extinction coefficient $\left(\varepsilon_{280}=66,810 \mathrm{M}^{-1} \mathrm{~cm}^{-1}\right)$ calculated using the ProParam tool

(www.expasy.ch/tools/). The absorbance at $280 \mathrm{~nm}$ was corrected for the contribution due to free FAD in guanidine hydrochloride $\left(\varepsilon_{280}=22,900 \mathrm{M}^{-1} \mathrm{~cm}^{-1}\right)(9)$.

\section{Mass Spectral Analysis}

NikD ligands were released by acetonitrile denaturation of enzyme expressed using TB as the growth medium. LC/MS/MS analyses were conducted using a Waters Q-Tof micro ${ }^{\mathrm{TM}}$ Quadrupole Time-of-Flight mass spectrometer with a MassLynx workstation. Ligand extracts 
or standards were subjected to electrospray ionization (ES) and positive/negative switching. Except as noted below, all HPLC separations were carried out on an Alliance ${ }^{\mathrm{TM}}$ HPLC system using a Waters' Atlantis ${ }^{\mathrm{TM}} \mathrm{dC} 18$ column $(3 \mu \mathrm{m}$ particle, $2.1 \mathrm{~mm} \times 150 \mathrm{~mm})$ and method 4 (flow rate: $250 \mu \mathrm{L} / \mathrm{min}$ ) at $20{ }^{\circ} \mathrm{C}$. Typical settings were: cone voltage, $20 \mathrm{eV}$; collision energy, $1 \mathrm{eV}$ (ESI-MS) or 10, 15, 20 (ESI-MS-MS); argon, 13 psi; source, $125^{\circ} \mathrm{C}$; desolvation, $290{ }^{\circ} \mathrm{C}$. For studies on compound $\mathrm{III}_{\mathrm{TB}}$ in ligand extracts, HPLC separations were conducted using an ACQUITY UPLC ${ }^{\mathrm{TM}}$ system operated in HPLC mode using a Hamilton PRP-X100 anion exchange column $(5 \mu \mathrm{m}$ particle, $4.6 \mathrm{~mm} \times 250 \mathrm{~mm}$ ) and method 2 (flow rate: $1.2 \mathrm{~mL} / \mathrm{min}$ ). Typical settings were: cone voltage, $20,50 \mathrm{~V}$; argon, 13 psi; source, $130{ }^{\circ} \mathrm{C}$; desolvation, 300 ${ }^{\circ} \mathrm{C}$. The following tolerance limits were imposed in conducting single mass analyses to identify possible molecular species corresponding to a specified $\mathrm{m} / \mathrm{z}$ value: tolerance, $11.0 \mathrm{ppm}$; double bond equivalents $(\mathrm{DBE}), \min =0.0, \max =25.0$; isotope cluster parameters, separation $=1.0$, abundance $=1.0 \%$.

\section{Preparation of Ligand-depleted NikD}

All steps were conducted at 0 to $4{ }^{\circ} \mathrm{C}$. A solution containing $37 \mathrm{mg}$ of nikD in $10 \mathrm{~mL}$ of 50 $\mathrm{mM}$ potassium phosphate buffer, $\mathrm{pH} 8.0$, (buffer $\mathrm{A}$ ) was mixed at approximately $30 \mathrm{~s}$ intervals with $0.5 \mathrm{~mL}$ aliquots of buffer A containing $2 \mathrm{mM}$ EDTA and $4 \mathrm{M}$ guanidine hydrochloride until a final guanidine hydrochloride concentration of $2 \mathrm{M}$ was reached. The sample was centrifuged after a 30 min incubation on ice and then dialyzed overnight against buffer A containing $1 \mathrm{mM}$ EDTA and $2 \mathrm{M}$ guanidine hydrochloride. The guanidine hydrochloride concentration was then gradually decreased by dialysis for $2 \mathrm{~h}$ against buffer A containing 1 mM EDTA and 2.0, 1.5, 1.0, $0.75,0.50$ or $0.25 \mathrm{M}$ guanidine hydrochloride. The sample was then dialyzed overnight against buffer $\mathrm{A}$, followed by a $2 \mathrm{~h}$ dialysis against buffer $\mathrm{A}$. Spectral and activity measurements were performed after concentrating the solution to $1.5 \mathrm{~mL}$ using a Centriprep 30. A heat extract of the refolded protein was prepared and subjected to HPLC analysis, as described above.

\section{Mutation of Trp355 to Phe}

All PCR reactions were conducted using a Hybaid Touchdown Thermocycler. Unless otherwise noted, PCR products were purified by agarose gel (1.5\%) electrophoresis and recovered using a QIAQuick Gel Extraction Kit (Qiagen). Sequencing was conducted by MWG Biotech. Mutations were generated by using the plasmid pDV101 (5) as template and the overlap extension PCR method described by Ho et al. (10). PCR reactions were performed by using Pfu DNA polymerase (Stratagene) and the following settings: 1 cycle of $94^{\circ} \mathrm{C}$ for $2 \mathrm{~min}$; 30 cycles of $94{ }^{\circ} \mathrm{C}$ for $40 \mathrm{~s}, 52{ }^{\circ} \mathrm{C}$ for $40 \mathrm{~s}, 72^{\circ} \mathrm{C}$ for $2 \mathrm{~min} ; 1$ cycle, $72{ }^{\circ} \mathrm{C}$ for $30 \mathrm{~min}$; 1 cycle, $30{ }^{\circ} \mathrm{C}$ for $5 \mathrm{~min}$. The left-hand fragment was generated using $5^{\prime}$ -

GACTCACTATAGGGAGACCACAAC-3' (START) as forward primer and 5'-

GAACTTGAACGCatAGCCCGCCCC-3' as backward primer. The right-hand fragment was generated using 5'-GGGGCGGGCTatGCGTTCAAGTTC-3' as forward primer and 5'CCGGATATAGTTCCTCCTTTCAGC-3' (END) as backward primer. The purified left- and right-hand fragments were combined using START and END as forward and backward primers, respectively. (Mutagenic sites in the primers are shown in lower case; codon 355 is underlined.) The final PCR product was purified by using a QIAquick PCR Purification Kit (Qiagen), digested with NdeI and XhoI, purified again and then subcloned between the NdeI and XhoI sites of plasmid pET23a. The resulting construct was used to transform E. coli BL21 (DE3) cells to ampicillin resistance. For screening, plasmid DNA was isolated from randomly selected clones using the QiaPrep Spin Miniprep kit (Qiagen) and digested with NdeI and XhoI. A plasmid that exhibited the expected insert size (pGZ42) was isolated using the Qiagen Plasmid Midi kit (Qiagen) and sequenced across the entire insert. 


\section{RESULTS}

\section{Endogenous Ligands in NikD Extracts}

We expected that nikD extracts would contain a single compound corresponding to the endogenous ligand observed at the active site in the crystal structures. Instead, extracts prepared by denaturation with heat or acetonitrile were found to contain at least four different compounds, as judged by HLPC analysis using an anion exchange or a reversed phase column (Figures 2 and 3). As described below, compounds I and IV are structurally related and share a common binding site whereas compound III binds at a different site. Compound II is a decomposition product of compound I.

\section{Identification of Compound II}

The following observations indicate that compound II is AMP. Compound II and AMP exhibit identical HPLC elution profiles (Figures 2 and 3$)$ and absorption spectra $\left(\lambda_{\max }=259 \mathrm{~nm}\right.$, data not shown). Both compounds are converted to adenosine upon treatment with alkaline phosphatase (data not shown) and exhibit the same molecular weight (347 Da), as judged by mass spectral analysis in positive or negative mode (Table I).

\section{Properties and Identification of Compounds I and IV as Derivatives of ADP}

Compound I is labile. Quantitative hydrolysis to AMP is observed after a $1 \mathrm{~h}$ incubation in 100 $\mathrm{mM} \mathrm{KOH}$ at room temperature (data not shown, see Supporting Information Figure S1). Compound I elutes as a single broad peak from an anion exchange HPLC column (Figure 2) whereas a pair of closely eluting peaks (Ia, Ib) are observed in the reversed phase elution profile (Figure 3). Mass spectral analysis in combination with reversed phase HPLC shows that compounds Ia and $1 \mathrm{~b}$ exhibit the same molecular weight (619 Da, Table I) and thus appear to be isomers.

Unlike compound I, compound IV is stable under basic conditions and not easily recovered from an anion exchange column. However, compound IV is readily isolated by reversed phase HPLC (Figure 3). The purified compound exhibits an absorption spectrum $\left(\lambda_{\max }=259 \mathrm{~nm}\right)$ that is virtually identical to that of AMP (data not shown). Compound IV is more than twice the size of compound I, as judged by the molecular weight determined by mass spectral analysis (1372 Da, Table 1).

MS/MS analyses in positive ion mode were conducted to characterize fragments derived from compounds I and IV. Signals attributable to AMP, the adenine moiety of AMP (Ade) and a phosphorylated derivative of AMP were observed in MS/MS ES+ spectra obtained with both compounds I and IV (data not shown, see Supporting Information Figures S2 and S3, respectively). Phosphorylation of the 5'-phosphate in AMP would produce an ADP derivative that should be susceptible to hydrolysis by phosphodiesterase I, unlike compounds generated by phosphorylation at other possible sites (e.g., 2'-OH or 3'-OH in AMP). Indeed, quantitative hydrolysis of compounds Ia, Ib and IV to AMP is observed upon treatment of a crude ligand extract with purified phosphodiesterase I. No other UV-absorbing products are detected (Figure 4). The results indicate that compounds I and IV are ADP derivatives.

\section{Quantitative Analysis of Compounds I and IV in NikD Preparations}

Compounds I and IV are too big to fit into the active site of nikD but might be expected to bind at the dinucleotide binding motif in the flavin domain, in competition with each other and FAD. In this case, the sum of all three compounds should not exceed one mol per mol of nikD. To test this hypothesis, quantitative analyses were conducted with extracts from three enzyme preparations that contained between 0.54 to $0.82 \mathrm{~mol}$ of FAD. (The FAD content was determined separately, as described in Experimental Procedures, because the covalently bound 
flavin is not released into the extract obtained upon enzyme denaturation.) The amount of compound I varied between 0.02 to $0.14 \mathrm{~mol}$ per mol of nikD and was not correlated with the FAD content. In contrast, the amount of compound IV was inversely proportional to the flavin content $\left(\mathrm{r}^{2}=0.9589\right)$ and varied between 0.12 to $0.45 \mathrm{~mol}$ per mol of nikD. The molar sum of FAD, compound I and compound IV in the three preparations varied between 0.98 to $1.05 \mathrm{~mol}$ per mol of nikD (Table 2). The results support the proposal that all three compounds compete for a common ADP-binding site in nikD.

\section{Characterization of Compound III}

Since the other ligands could be ruled out, compound III emerged as a likely candidate for the ligand found at the active site in nikD crystals. Two different forms of compound III are found in nikD extracts, depending on whether the enzyme is expressed in cells grown in LB (compound $\mathrm{III}_{\mathrm{LB}}$ ) or Terrific Broth (TB) (compound $\mathrm{III}_{\mathrm{TB}}$ ). Neither compound acts as a substrate for phosphodiesterase I. Both compounds are difficult to elute from a reversed phase column but exhibit well-defined elution profiles from an anion exchange column (Figure 2).

The active site ligand in nikD crystals is modeled as an aromatic carboxylate containing a 6membered ring. Compounds that satisfy this criterion were subjected to HPLC analysis using an anion exchange column. Identical elution profiles are obtained with compound $\mathrm{III}_{\mathrm{LB}}$ and picolinate in analyses conducted by using a high resolution solvent system capable of separating picolinate from its positional isomers, nicotinate and isonicotinate (6) (Figure 2). Significantly, picolinate is the product of the reaction catalyzed by nikD with its physiological substrate (Scheme 1). A sub-stoichiometric amount of picolinate is found in nikD, as judged by quantitative HPLC analyses with extracts from two different preparations of enzyme that had been expressed in LB $(0.069 \pm 0.005 \mathrm{~mol}$ picolinate $/ \mathrm{mol}$ protein). The modest amount of picolinate detected in the isolated enzyme is consistent with the observed stability of the nikD.picolinate complex $\left(\mathrm{K}_{\mathrm{d}}=300 \mu \mathrm{M}\right)(5)$. Although picolinate is largely replaced by compound $\mathrm{III}_{\mathrm{TB}}$ in extracts from enzyme expressed in $\mathrm{TB}$, these extracts do contain a small amount of picolinate, as judged by results obtained in a preparative scale isolation of compound $\mathrm{III}_{\mathrm{TB}}$ (data not shown).

Compound $\mathrm{III}_{\mathrm{TB}}$ exhibits a broad, featureless absorption band in the UV region with a maximum at $265 \mathrm{~nm}$, unlike the relatively narrow, resolved peak observed with picolinate $\left(\lambda_{\max }=264 \mathrm{~nm}\right)($ data not shown, see Supporting Information Figure S4). HPLC analyses show that compound $\mathrm{III}_{\mathrm{TB}}$ is readily distinguishable from picolinate (Figure 2) and all of the other aromatic carboxylates surveyed, including nicotinate, isonicotinate, benzoate, 2-pyrazine carboxylate, 2- pyrimidinecarboxylate and 4-pyrimdinecarboxylate (data not shown). A signal attributable to compound $\mathrm{III}_{\mathrm{TB}}$ was not detected upon mass spectral analysis in positive or negative mode. An appropriate signal was obtained in control studies with picolinate.

\section{Preparation and Characterization of Ligand-depleted NikD}

Compounds I and IV do not bind at the active site and therefore cannot act as the intrinsic charge transfer donor. Compound $\mathrm{III}_{\mathrm{LB}}$ (picolinate) does bind at the active site. However, picolinate cannot be the intrinsic charge transfer donor because binding of this competitive inhibitor is accompanied by a loss of the enzyme's charge transfer band (5) (see inset to Figure $5 \mathrm{~A}$ ). To determine whether compound $\mathrm{III}_{\mathrm{TB}}$ might act as the intrinsic charge transfer donor, we developed a method for removing the endogenous ligands from enzyme that had been expressed in TB.

A dilute solution of nikD was slowly unfolded at $0{ }^{\circ} \mathrm{C}$ by step-wise addition of guanidine hydrochloride to a final concentration of $2 \mathrm{M}$. The sample was then dialyzed against buffer containing $2 \mathrm{M}$ guanidine hydrochloride to remove any ligands that had been released from 
the protein. The concentration of guanidine hydrochloride was then gradually decreased in a series of $2 \mathrm{~h}$ dialyses against solutions containing a progressively lower concentration of denaturant. This approach resulted in $42 \%$ recovery of the starting material as a soluble protein. The refolded preparation and untreated nikD exhibit similar specific activity values (24.0 and $22.4 \mathrm{~min}^{-1}$, respectively). Importantly, the absorption spectrum of the refolded enzyme displays a long wavelength absorption band identical to that observed with untreated enzyme (Figure 5A). The ligand content of the refolded enzyme is greatly reduced, as judged by the amount of UV-absorbing material recovered upon heat denaturation (Figure 5B). A heat extract of the refolded enzyme was subjected to HPLC analysis using anion exchange and reversed phase columns. Compound $\mathrm{III}_{\mathrm{TB}}$ was not detected. The extract did contain a small residual amount of the ADP derivatives and AMP (data not shown). The results show that compound $\mathrm{III}_{\mathrm{TB}}$ does not act as the intrinsic charge transfer donor.

\section{Mutation of Trp355}

Since all of the endogenous ligands could be ruled out, the intrinsic charge transfer donor must be an amino acid residue. Trp355 appeared to be the most likely candidate as judged by the crystal structure of the open form of nikD where the indole ring of Trp355 is stacked ( $3.5 \AA$ ) above the flavin ring. Accordingly, a conservative Phe mutation was introduced at Trp355. The Trp355Phe mutant was expressed in LB medium at $22{ }^{\circ} \mathrm{C}$ and purified by using a $\mathrm{Ni}^{2+}$ affinity matrix to apparent homogeneity, as judged by SDS-PAGE. We obtained $19 \mathrm{mg}$ of protein from $5 \mathrm{~L}$ of cell culture, a yield that is about $50 \%$ of that observed with wild type nikD (5). The mutant protein contained $0.42 \mathrm{~mol}$ of covalently bound FAD, a value somewhat lower than observed with various preparations of wild type enzyme (Table 3).

Importantly, the Trp355Phe mutant exhibits a typical flavin absorption spectrum with no evidence for a long wavelength charge transfer band (Figure 6). The results provide definitive evidence for Trp355 as the intrinsic charge transfer donor in wild type nikD. The mutation also causes a bathochromic shift of the unresolved absorption band that is observed with wild type enzyme at $455 \mathrm{~nm}$. In the mutant enzyme, this band is shifted to $462 \mathrm{~nm}$ and is highly resolved with a pronounced shoulder at $485 \mathrm{~nm}$, suggesting a less polar flavin environment than in wild type nikD (11).

\section{Catalytic Properties of the Trp355Phe Mutant}

The specific activity of the mutant is about $10 \%$ of that observed with wild type nikD, as judged by results obtained using a standard assay that contains $50 \mu \mathrm{M}$ P2C (Table 3). Although this substrate concentration is saturating for wild type nikD, steady-state kinetic studies indicate that it is not saturating for the mutant protein which exhibits an apparent $\mathrm{K}_{\mathrm{m}}$ that is 30 -fold larger than wild type nikD. In contrast, the mutation causes only a 3-fold decrease in the apparent $\mathrm{k}_{\text {cat }}$ value (Table 3).

The results suggest that the Trp355Phe mutation primarily affects substrate binding. Evidence to evaluate this hypothesis was sought by measuring the binding affinity of a substrate analog to the mutant enzyme. Dissociation constants for various inhibitor complexes are readily measured with wild type nikD by monitoring the accompanying perturbation of the visible absorption spectrum of the enzyme. Various attempts to conduct similar studies with the Trp355Phe mutant revealed that the mutation caused a significant decrease in enzyme stability, as judged by the observed aggregation under conditions ( $\mathrm{pH} 8.0,25$ or $5{ }^{\circ} \mathrm{C}$ ) where the wild type enzyme is stable for prolonged periods. As an alternate approach, we compared the effect of 1-cyclohexenoate, a 1-deaza analog of P2C, on turnover of mutant and wild type enzymes. In each case, 1-cyclohexenoate was found to act as a competitive inhibitor with respect to P2C (data not shown, see Supporting Information Figure S5). The inhibition constant with the 
mutant enzyme is 35 -fold larger than with wild type nikD (Table 3), consistent with the postulated role of Trp355 in substrate binding.

\section{DISCUSSION}

NikD crystals contain an endogenous active site ligand, modeled as an aromatic carboxylate with a 6-membered ring (7). Unexpectedly, nikD extracts have been found to contain multiple endogenous ligands (compounds I-IV). Compound II (AMP) is a decomposition product of compound I. Compounds I (619 Da) and IV (1372 Da) are ADP derivatives that bind to the dinucleotide binding motif in the enzyme's flavin domain, in competition with each other and the natural FAD prosthetic group. Consistent with this proposal, the molar sum of all three ADP derivatives exhibits a 1:1 stoichiometry with respect to nikD. Although nikD preparations contain variable amounts of FAD, the ADP-binding site in nikD crystals is fully occupied by FAD (7), suggesting that substitution of FAD by compound I or IV interferes with enzyme crystallization. Covalent attachment of FAD is probably "catalyzed" by the nikD apoprotein, as judged by results obtained with the homologous monomeric sarcosine oxidase (12). Misfolding of the nikD apoprotein might therefore promote adventitious binding of ADP metabolites by blocking covalent flavin incorporation. Alternatively, properly folded apoprotein might accumulate and bind ADP derivatives because overexpression of the recombinant enzyme has outstripped the cellular capacity for flavin biosynthesis.

Compound $\mathrm{III}_{\mathrm{LB}}$ is found in extracts from enzyme expressed in LB. This compound has been identified as picolinate, the product of the physiological catalytic reaction. Picolinate is also detectable in extracts from enzyme expressed in TB but largely replaced by a picolinate-like compound of currently unknown structure (compound $\mathrm{III}_{\mathrm{TB}}$ ). Picolinate matches the properties deduced for the active site ligand in crystals which have been obtained using enzyme expressed in either LB or TB. Less than $0.1 \mathrm{~mol}$ of picolinate is found in enzyme expressed in LB whereas the corresponding crystals show that the active site is fully occupied by the endogenous ligand. We considered the possibility that the active site ligand might be a contaminant in the crystallization buffers but HPLC analysis failed to detect any UV-absorbing (aromatic) compounds in these reagents. We tentatively conclude that the observed difference in ligand content of the isolated enzyme as compared with crystalline nikD is due to selective crystallization of the enzyme-picolinate complex.

NikD exhibits a long wavelength absorption band extending out to nearly $700 \mathrm{~nm}$. This unusual feature has been attributed to an intrinsic charge transfer complex containing oxidized FAD as the charge transfer acceptor (5). Trp355 and the endogenous active site ligand emerged as likely candidates for the intrinsic charge transfer donor, based on crystal structures observed for open and closed forms of nikD, respectively (see Figure 1). Conversion of the free enzyme to a complex with picolinate is observed in the presence of excess picolinate $\left(\mathrm{K}_{\mathrm{d}}=300 \mu \mathrm{M}\right)$ but results in loss of the long wavelength absorption band (5), a feature that rules out compound $\mathrm{III}_{\mathrm{LB}}$ as the intrinsic charge transfer donor. Reversible unfolding of nikD in guanidine hydrochloride removes compound $\mathrm{III}_{\mathrm{TB}}$ and most of the adenine-containing endogenous ligands found in enzyme that has been expressed in TB. The refolded enzyme is catalytically active and retains the long wavelength absorption band, ruling out compound $\mathrm{III}_{\mathrm{TB}}$ as the intrinsic charge transfer donor. Importantly, the charge transfer band is eliminated by mutation of Trp355 to Phe. The results provide definitive evidence for Trp355 as the intrinsic charge transfer donor.

To our knowledge, nikD is the first example where a wild type flavoenzyme has been demonstrated to contain a flavin-tryptophan charge transfer complex. In flavodoxins, the outer and inner faces of the FMN ring are typically flanked by tyrosine and tryptophan residues, respectively. The tryptophan ring is inclined at an angle of approximately $20-45^{\circ}$ with respect 
to the flavin ring, a geometry unfavorable for charge transfer interaction. The tyrosine residue is, however, nearly coplanar with the flavin ring and a charge transfer complex is observed when this residue is mutated to tryptophan $(13,14)$. Similarly, a C-terminal tyrosine in ferredoxin-NADP ${ }^{+}$reductase (FNR) lies parallel to the flavin ring. Changing this tyrosine to tryptophan also yields a mutant enzyme that exhibits that exhibits a long wavelength charge transfer band (15). Although not established experimentally, a long wavelength absorption band exhibited by the FMN domain of cytochrome P450BM-3 may reflect charge transfer interaction between the flavin ring and a nearby, coplanar tryptophan residue $(16,17)$.

The identification of Trp355 as the charge transfer donor in nikD permits correlation between solution and crystalline forms. A diagnostic long wavelength absorption band is formed upon partial $\pi-\pi$ charge transfer from Trp355 to FAD in a process promoted by parallel stacking of the indole and flavin rings, a feature observed only in the open crystal form. Solutions of nikD exhibit a charge transfer band at slightly alkaline $\mathrm{pH}\left(\mathrm{pK}_{\mathrm{a}}=7.3\right)$ but not at slightly acidic $\mathrm{pH}$ and/or in the presence of active site ligands. This suggests that the equilibrium between the two conformations lies in favor of the open form in solution at slightly alkaline $\mathrm{pH}$. Conversely, the closed form predominates at slightly acidic $\mathrm{pH}$ and/or in the presence of active site ligands.

Substrate access to the active site in nikD is possible only in the open conformation but substrate oxidation is likely to require stacking of the substrate and flavin rings, as observed only in the closed conformation. A possible resolution of this paradox is provided by a two-step mechanism for substrate binding (Scheme 2). We postulate that an initial redox-inactive ES complex is produced when substrate binds to the open conformation. This solution complex is metastable but otherwise resembles the open crystal form of the nikD complex with picolinate. In solution, the presence of bound ligand shifts the equilibrium in favor of the closed conformation, producing a redox-active ES complex. We suggest that the crystalline environment may stabilize the crystal in an open, ligand-bound form.

Mutation of Trp355 in nikD to Phe results in a 30-fold increase in the apparent $\mathrm{K}_{\mathrm{m}}$ for P2C and a 35-fold increase in the $\mathrm{K}_{\mathrm{i}}$ for 1-cyclohexenoate, a 1-deaza analog of P2C. The results indicate that Trp355 exerts a net positive effect on substrate binding even though formation of a redox-active ES complex is likely to involve displacement of Trp355 (Scheme 2). The energetic cost of Trp355 displacement may be compensated by favorable interactions in the closed form where Trp355 constitutes part of an aromatic cage that surrounds the ligand ring and also forms a hydrogen bond with the side chain of Asn100 (Figure 1). The absorption spectrum of the Trp355Phe mutant is strikingly similar to that observed with the closed form of wild type enzyme. This suggests that the mutation may shift the equilibrium towards the closed form, a feature that could contribute to the observed decrease in substrate binding affinity. Substrate-induced displacement of an aromatic residue has been observed with at least two other flavoenzymes, including FNR and glutathione reductase. Unlike nikD, the aromatic residue in FNR (Tyr308) interferes with substrate binding but is, nevertheless, found to facilitate catalysis, probably by accelerating product release $(15,18)$. The aromatic residue in glutathione reductase (Tyr197) is thought to assist catalysis by acting as a spring that presses the nicotinamide ring in NADPH against the flavin ring (19).

\section{Supplementary Material}

Refer to Web version on PubMed Central for supplementary material.

\section{Acknowledgments}

We thank the Waters Corporation (Mildford, MA 01757) for the use of their facilities and Doug Stevens and Andy Whitehill for their expert technical assistance in the mass spectral analysis of nikD ligands. 


\section{References}

1. Fiedler HP, Kurth R, Langharig J, Delzer J, Zahner H. Nikkomycins: Microbial inhibitors of chitin synthetase. J Chem Biotechnol 1982;32:271-280.

2. Hector RF. Compounds active against cell walls of medically important fungi. Clin Microb Rev 1993;6:1-21.

3. Bruntner C, Bormann C. The Streptomyces tendae Tu901 L-lysine 2-aminotransferase catalyzes the initial reaction in nikkomycin D biosynthesis. Eur J Biochem 1998;254:347-355. [PubMed: 9660190]

4. Bruntner C, Lauer B, Schwarz W, Mohrle V, Bormann C. Molecular characterization of co-transcribed genes from Streptomyces tendae Tu901 involved in the biosynthesis of the peptidyl moiety of the peptidyl nucleoside antibiotic nikkomycin. Mol Gen Genet 1999;262:102-114. [PubMed: 10503541]

5. Venci D, Zhao G, Jorns MS. Molecular characterization of nikD, a new flavoenzyme important in the biosynthesis of nikkomycin antibiotics. Biochemistry 2002;41:15795-15802. [PubMed: 12501208]

6. Bruckner RC, Zhao G, Venci D, Jorns MS. Nikkomycin biosynthesis: Formation of a 4-electron oxidation product during turnover of nikD with its physiological substrate. Biochemistry 2004;43:9160-9167. [PubMed: 15248773]

7. Jorns, MS.; Bruckner, RC.; Zhao, G.; Carrell, CJ.; Mathews, FS. NikD: Crystal structures, charge transfer complex and endogenous ligands. In: Nishino, T.; Miura, R.; Tanokura, M.; Fukui, K., editors. Flavins and Flavoproteins 2005. ARchiTech Inc.; Tokyo: 2005. p. 773-785.

8. Willie A, Jorns MS. Discovery of a third coenzyme in sarcosine oxidase. Biochemistry 1995;34:1670316707. [PubMed: 8527444]

9. Wagner MA, Khanna P, Jorns MS. Structure of the flavocoenzyme of two homologous amine oxidases: Monomeric sarcosine oxidase and N-methyltryptophan oxidase. Biochemistry 1999;38:5588-5595. [PubMed: 10220347]

10. Ho SN, Hunt HD, Horton RM, Pullen JK, Pease LR. Site-directed mutagenesis by overlap extension using the polymerase chain reaction. Gene 1989;77:51-59. [PubMed: 2744487]

11. Harbury HA, LaNoue KF, Loach PA, Amick R. Molecular interaction of isoalloxazine derivatives. II. Proc Natl Acad Sci USA 1959;45:1708-1717. [PubMed: 16590562]

12. Hassan-Abdallah A, Bruckner RC, Zhao GH, Jorns MS. Biosynthesis of covalently bound flavin: Isolation and in vitro flavinylation of the monomeric sarcosine oxidase apoprotein. Biochemistry 2005;44:6452-6462. [PubMed: 15850379]

13. Swenson RP, Krey GD. Site-directed mutagenesis of tyrosine-98 in the flavodoxin from Desulfovibrio vulgaris (Hildenborough): Regulation of oxidation-reduction properties of the bound FMN cofactor by aromatic, solvent, and electrostatic interactions. Biochemistry 1994;33:8505-8514. [PubMed: 8031784]

14. Lostao N, Gomezmoreno C, Mayhew SG, Sancho J. Differential stabilization of the three FMN redox forms by tyrosine 94 and tryptophan 57 in flavodoxin from Anabaena and its influence on the redox potentials. Biochemistry 1997;36:14334-14344. [PubMed: 9398151]

15. Nogues I, Tejero J, Hurley JK, Paladini D, Frago S, Tollin G, Mayhew SG, Gomez-Moreno C, Ceccarelli EA, Carrillo N, Medina M. Role of the C-terminal tyrosine of ferredoxin-nicotinamide adenine dinucleotide phosphate reductase in the electron transfer processes with its protein partners ferredoxin and flavodoxin. Biochemistry 2004;43:6127-6137. [PubMed: 15147197]

16. Sevrioukova IF, Li H, Zhang H, Peterson JA, Poulos TL. Structure of a cytochrome P450-redox partner electron-transfer complex. Proc Natl Acad Sci 1999;96:1863-1868. [PubMed: 10051560]

17. Sevrioukova I, Truan G, Peterson JA. The flavoprotein domain of P450BM-3: Expression, purification, and properties of the flavin adenine dinucleotide- and flavin mononucleotide-binding subdomains. Biochemistry 1996;35:7528-7535. [PubMed: 8652532]

18. Deng Z, Aliverti A, Zanetti G, Arakaki A, Ottado J, Orellano EG, Calcaterra NB, Ceccarelli EA, Carrilli N, Karplus PA. A productive NADP(+) binding mode of ferredoxin-NADP(+) reductase revealed by protein engineering and crystallographic studies. Nature Structural Biology 1999;6:847853.

19. Karplus PA, Schulz GE. Substrate binding and catalysis by glutathione reductase as derived from refined enzyme-substrate crystal structures at 2 Å resolution. J Mol Biol 1989;210:163-180. [PubMed: 2585516] 

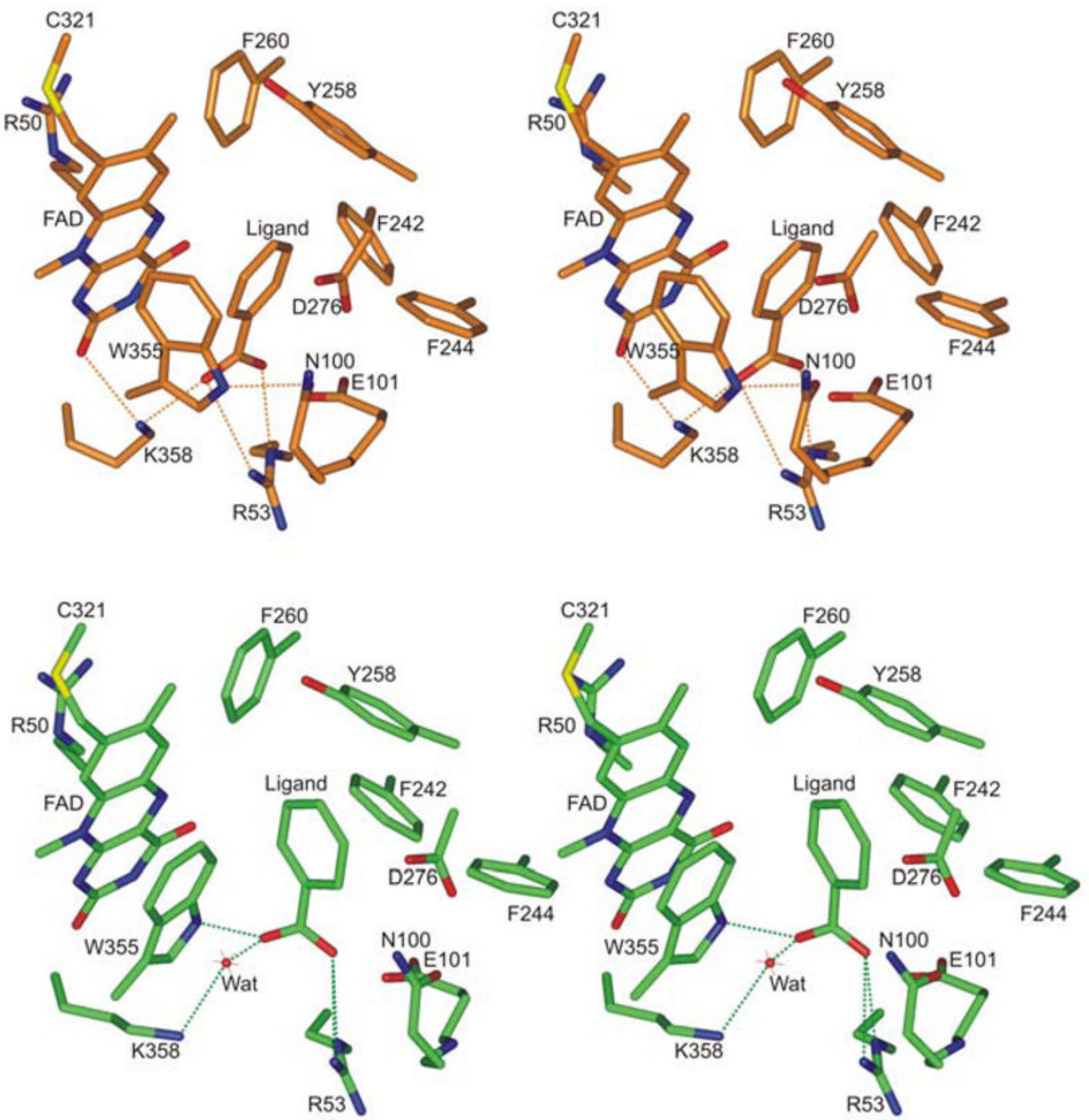

Figure 1.

Stereo views of the active site in the closed (top panel) and open (bottom panel) forms (PDB code $2 \mathrm{H} 2 \mathrm{~V}$ and $2 \mathrm{H} 2 \mathrm{~L}$, respectively). Atoms are colored: C, gold (closed form) or green (open form); O, red; N, blue; S, yellow. For clarity, only selected hydrogen bonds are shown. 

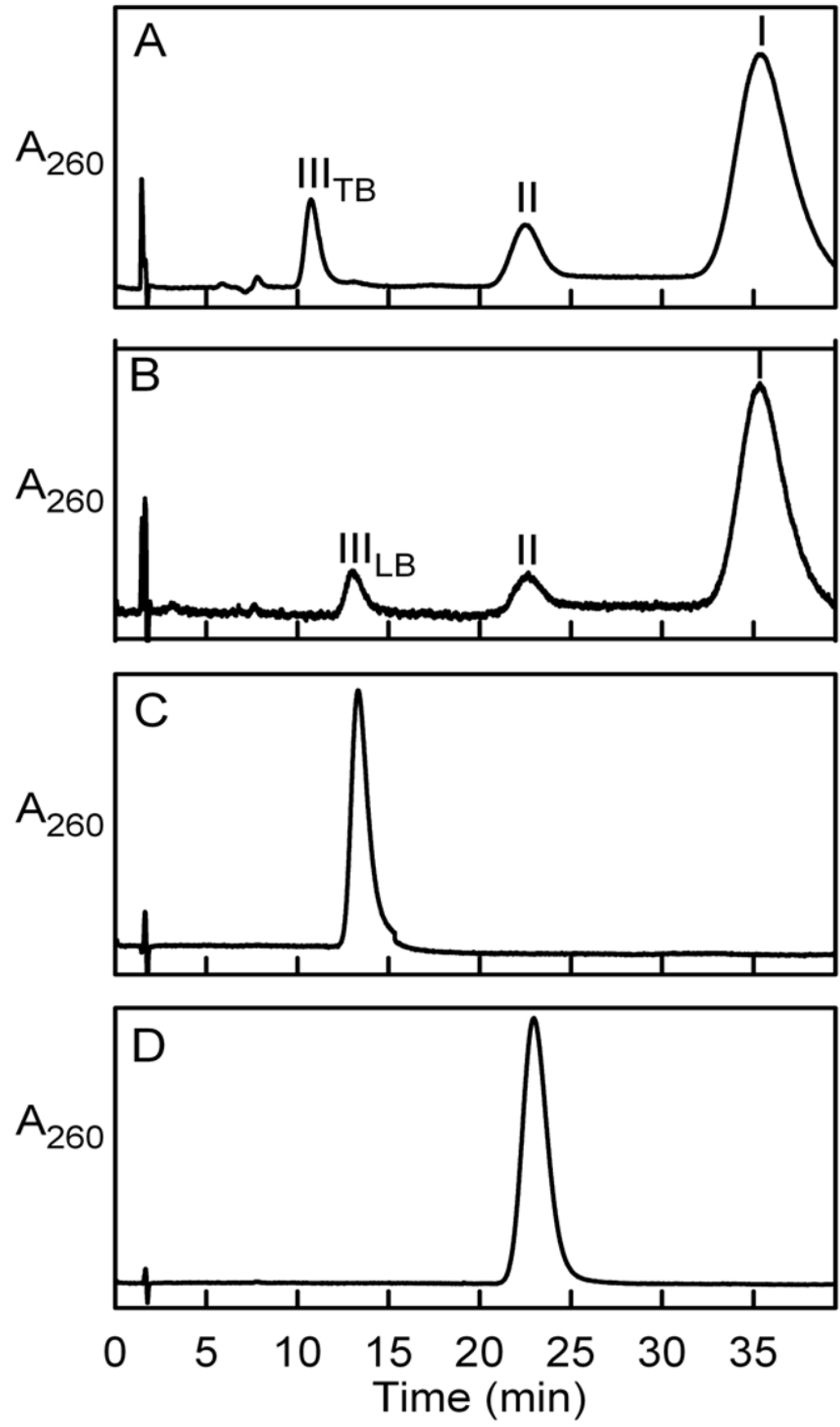

Figure 2.

Anion exchange HPLC analysis of nikD ligands. Extracts were prepared from nikD isolated from cells grown in TB (panel A) or LB (panel B) medium. Panels C and D are elution profiles obtained with picolinate and AMP, respectively. The chromatograms were developed using method 1. (Compound IV binds tightly to the anion exchange column and is not eluted under these conditions.) 

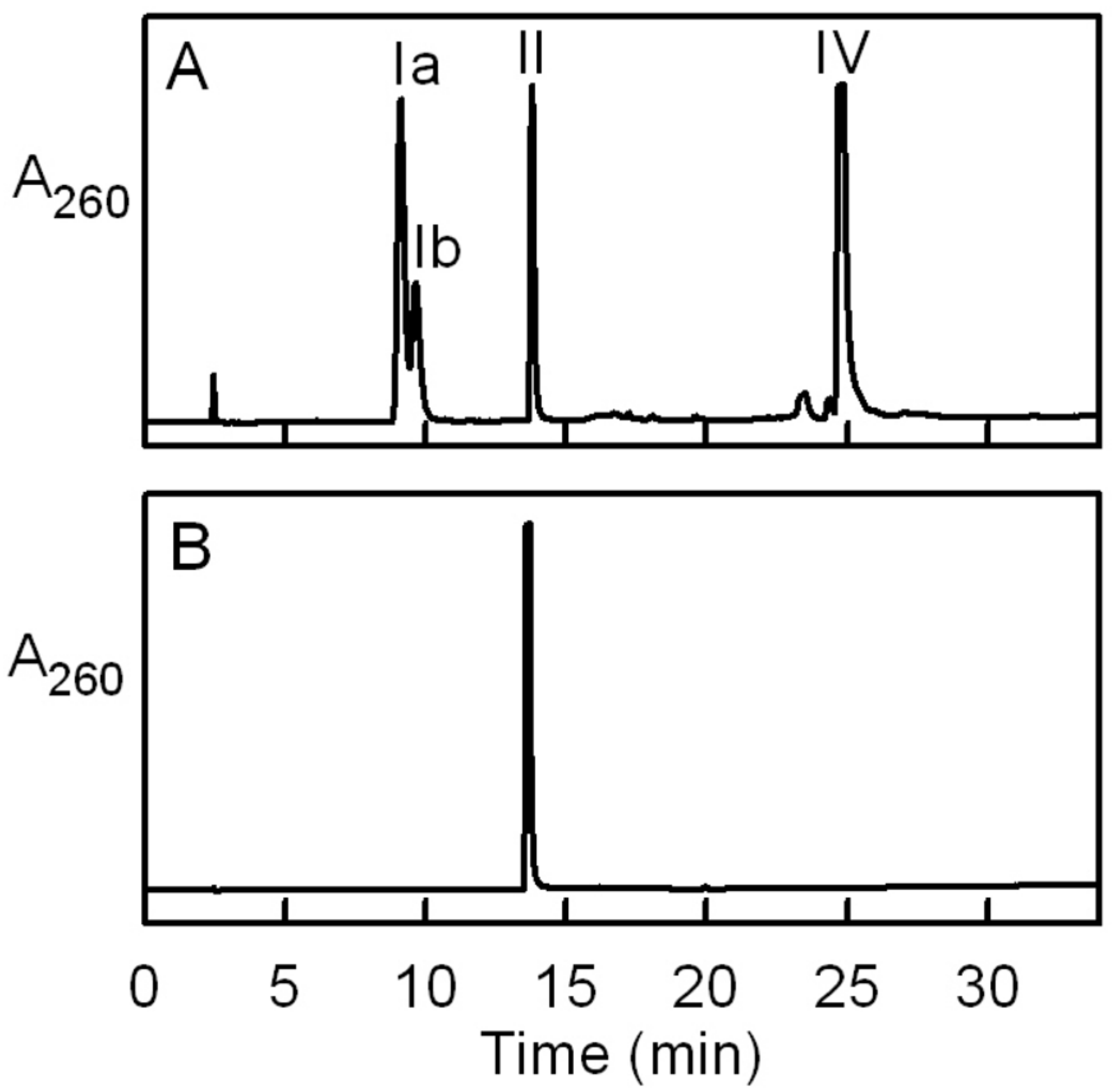

Figure 3.

Reversed phase HPLC analysis of nikD ligands. Panel A is the elution profile obtained with an extract prepared from nikD isolated from cells grown in TB medium. Panel B is the elution profile obtained with AMP. The chromatograms were developed using method 3. (Compound III is not eluted under these conditions.) 

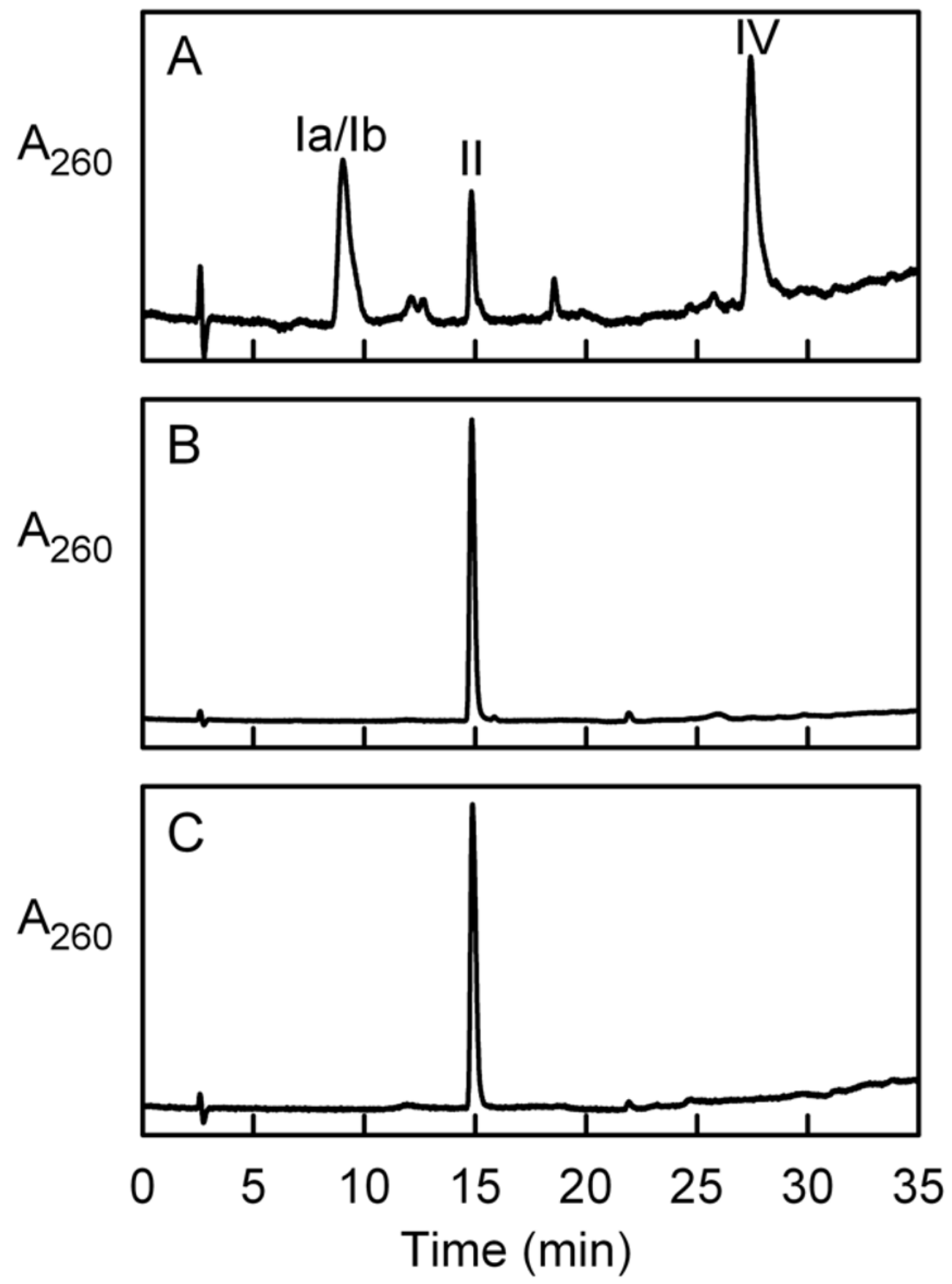

Figure 4.

Effect of phosphodiesterase I treatment on compounds I and IV. HPLC analyses were conducted using a reversed phase column and method 3. Panel A was obtained with an untreated ligand extract from enzyme that had been isolated from cells grown in TB medium. Panel B was obtained after incubating the extract with $0.0095 \mathrm{U} / \mathrm{mL}$ of purified

phosphodiesterase I for $60 \mathrm{~min}$ in $50 \mathrm{mM}$ potassium phosphate buffer, $\mathrm{pH} 8.8$ at $37{ }^{\circ} \mathrm{C}$. Panel C was obtained with AMP. 


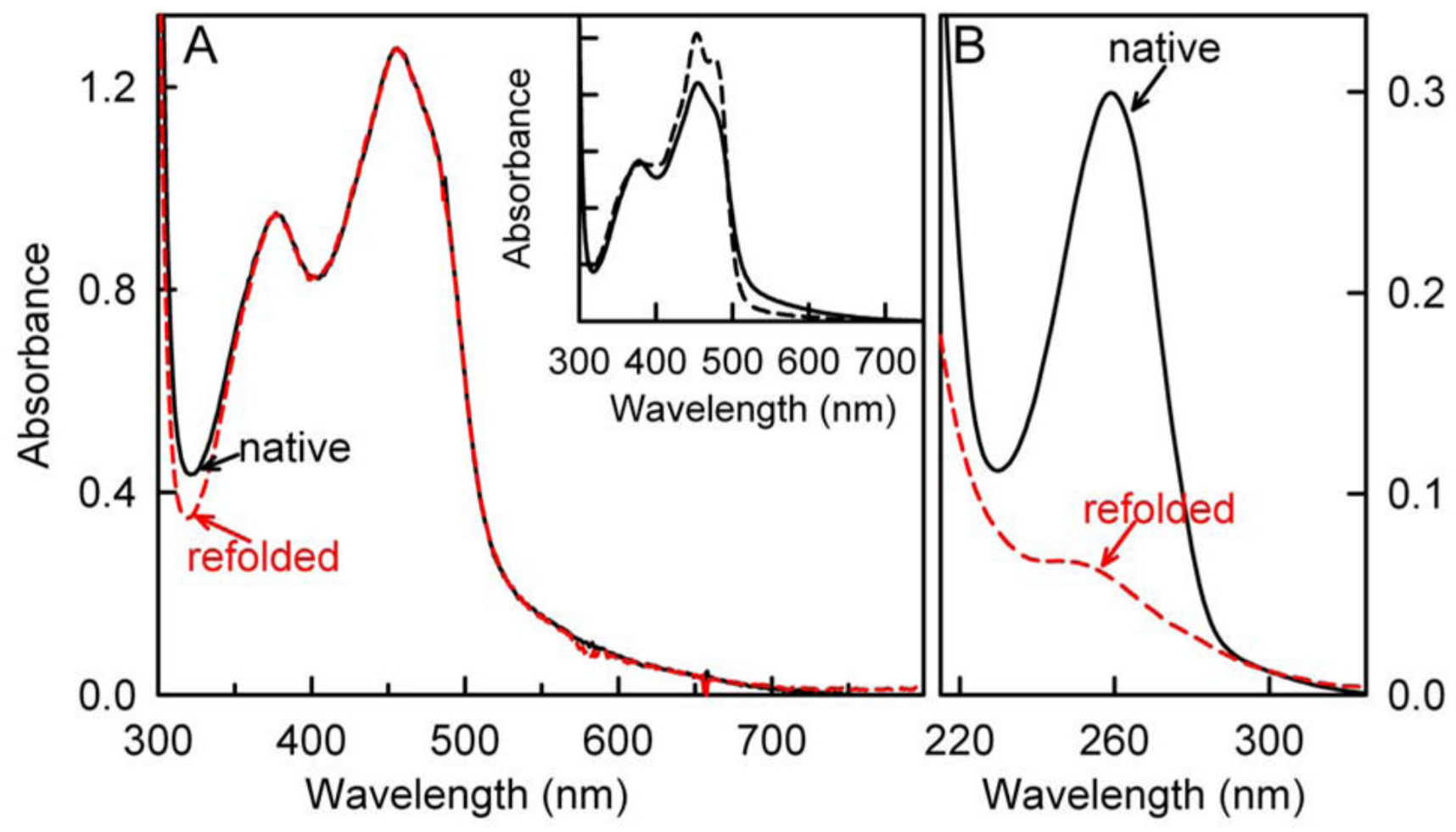

Figure 5.

Effect of unfolding and renaturation on the spectral properties and the endogenous ligand content of nikD. Panel A shows absorption spectra of native (solid black line) and refolded

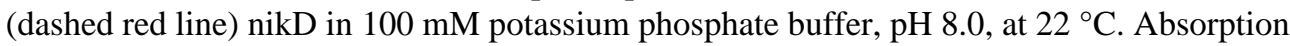
spectra for the corresponding heat extracts are shown in panel B. Spectra are normalized to the same enzyme concentration. The inset in panel A shows previously reported (5) absorption spectra of free nikD (solid line) and the enzyme-picolinate complex (dashed line) obtained at $25^{\circ} \mathrm{C}$ in $100 \mathrm{mM}$ potasium phosphate buffer, $\mathrm{pH} 8.0$, containing 0 and $2.27 \mathrm{mM}$ picolinate, respectively. 


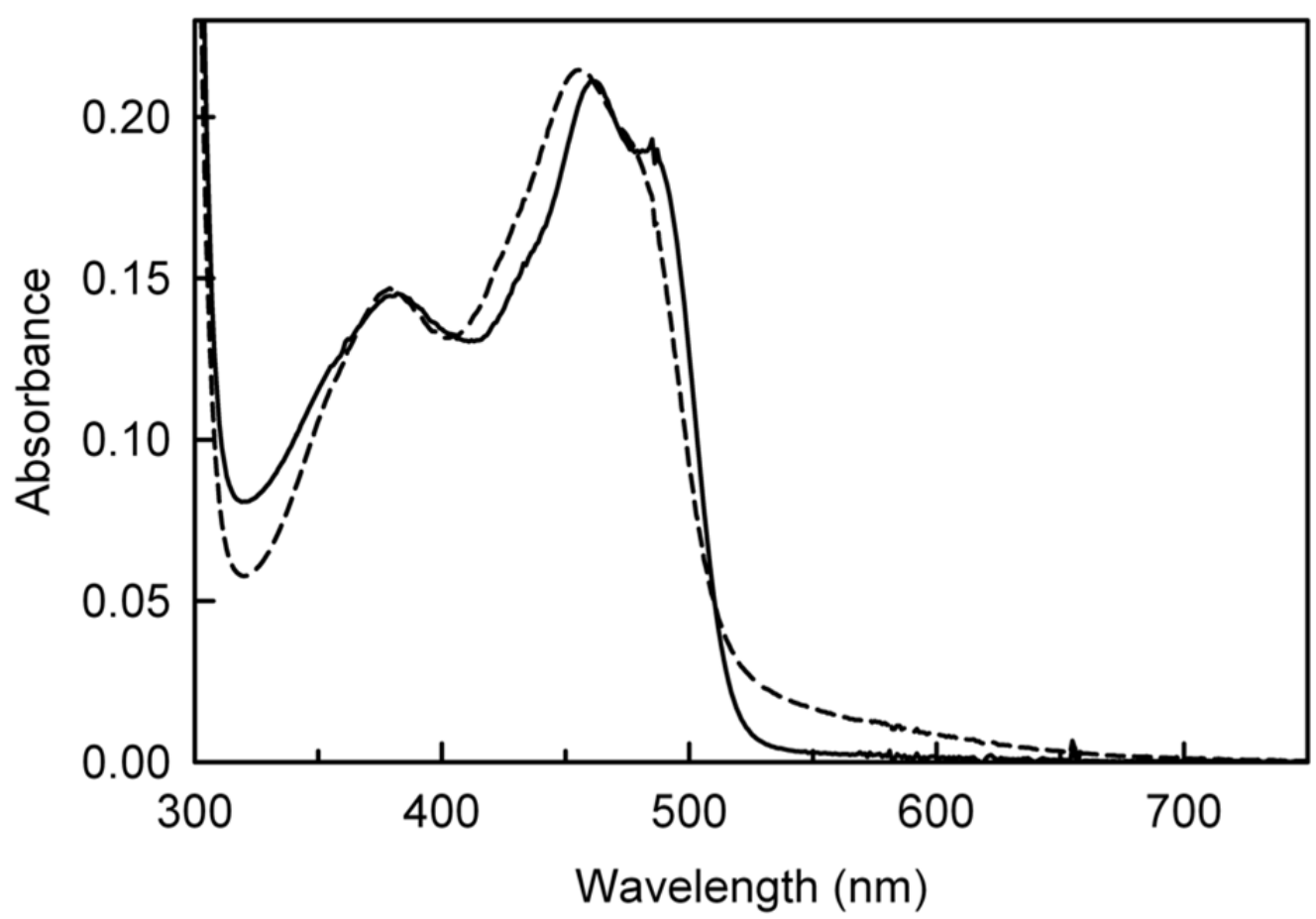

Figure 6.

Comparison of the spectral properties of the Trp355Phe mutant with wild type nikD. Spectra for the mutant (solid line) and wild type (dashed line) enzyme were recorded in $100 \mathrm{mM}$ potassium phosphate buffer, $\mathrm{pH} 8.0$ at $22{ }^{\circ} \mathrm{C}$. 


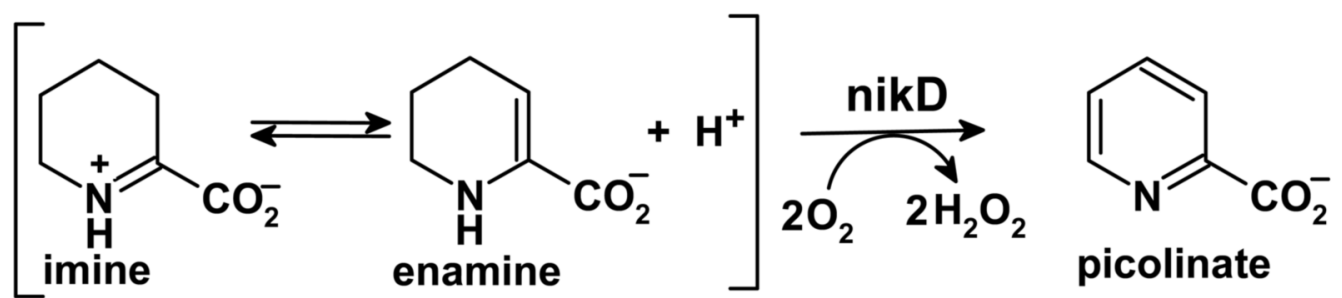

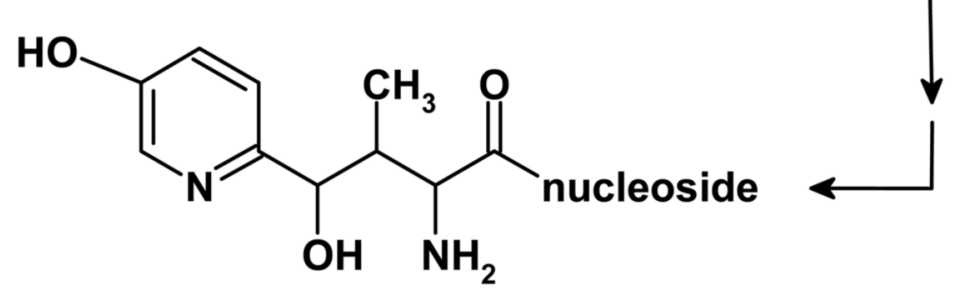
nikkomycin

Scheme 1.

Role of nikD in the biosynthesis of the peptidyl moiety of nikkomycins. 


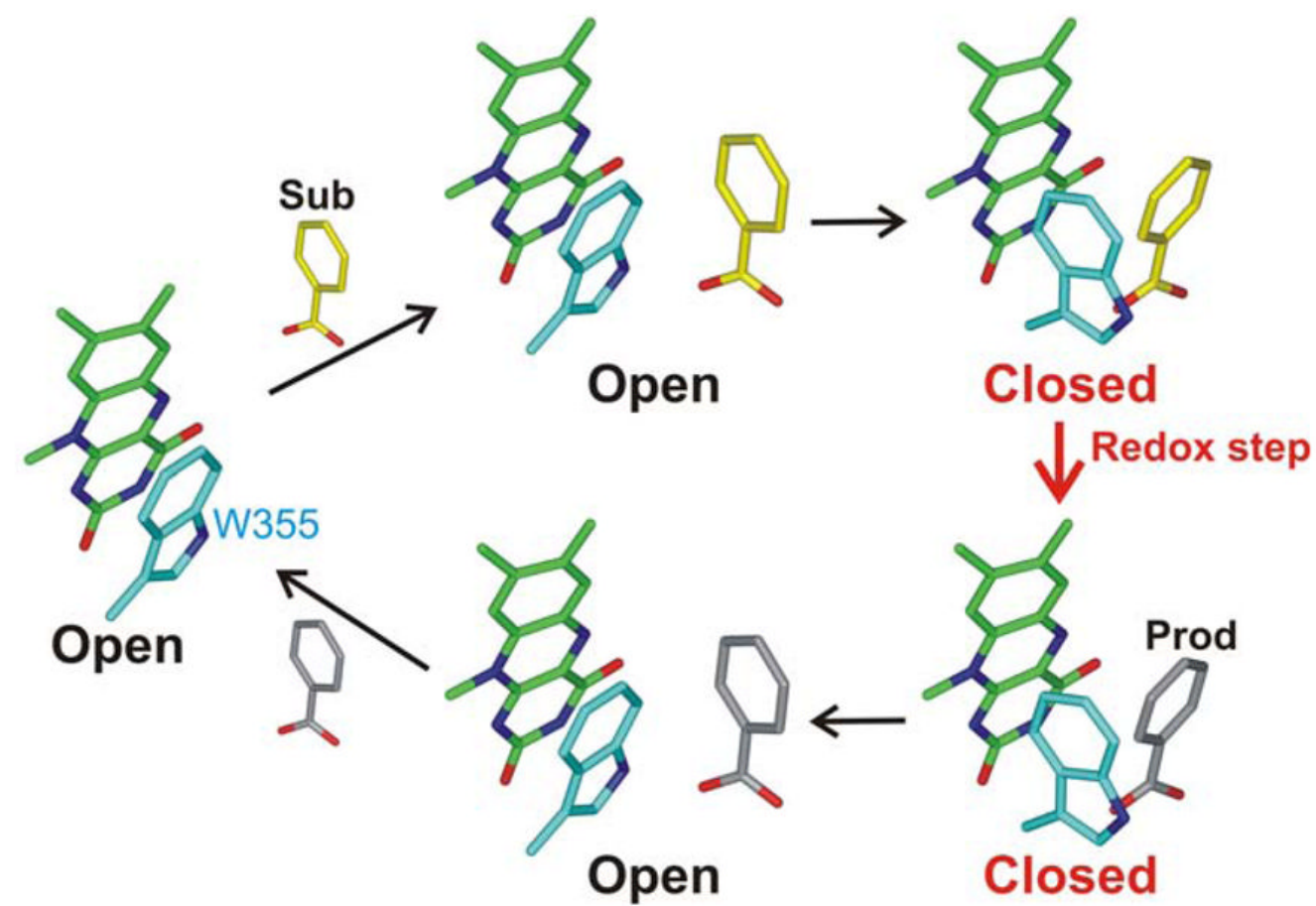

Scheme 2.

Postulated two-step mechanism for formation of a redox-active enzyme-substrate complex. 
Table 1

Mass Spectral Characterization of Compounds II, I and IV ${ }^{1}$

\begin{tabular}{lcc}
\hline & & $\mathbf{m} / \mathbf{z}$ \\
\cline { 2 - 3 } & $\mathbf{M}+\mathbf{H}$ & $\mathbf{M}-\mathbf{H}$ \\
\hline compound II & 348.0730 & 346.0570 \\
AMP (calc) & 348.0709 & 346.0553 \\
compound I & & 618.0835 \\
compound IV & 620.1053 & 1371.2270 \\
\hline
\end{tabular}

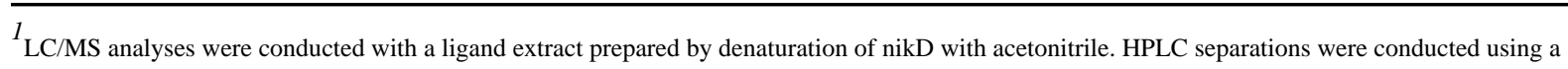
reversed phase column (method 4) connected to a Quadrupole Time-of-Flight mass spectrometer, as detailed in Experimental Procedures.

2 Identical results were obtained with compound Ia or Ib. 
Table 2

ADP derivatives in nikD preparations ${ }^{l}$

\begin{tabular}{lccc}
\hline Derivative & Prep A & mol derivative/mol nikD & prep B \\
& & 0.020 & 0.035 \\
\hline
\end{tabular}


Table 3

Comparison of the spectral and catalytic properties of Trp355Phe and wild type nikD

\begin{tabular}{|c|c|c|}
\hline & Trp355Phe & wild type \\
\hline absorption maxima $(\mathrm{nm})$ & 382,462 & $379,455^{a}$ \\
\hline absorption at $\lambda>550 \mathrm{~nm}$ & no & yes \\
\hline$\varepsilon_{4 \mathrm{xy}}\left(\mathrm{M}^{-1} \mathrm{~cm}^{-1}\right)^{b}$ & 12,700 & $11,200^{a}$ \\
\hline $\mathrm{A}_{280} / \mathrm{A}_{4 \mathrm{xy}} b$ & 13.6 & 8.77 to $13.0^{c}$ \\
\hline mol FAD/mol protein & 0.42 & 0.54 to $0.90^{c}$ \\
\hline specific activity $\left(\min ^{-1}\right)$ & 2.1 & $22.9 \pm 1.0^{d}$ \\
\hline $\mathrm{k}_{\text {cat (app) }}\left(\min ^{-1}\right)$ & $8.4 \pm 0.1$ & $28.2 \pm 0.8(37 \pm 0.9)^{e}$ \\
\hline $\mathrm{K}_{\mathrm{m}(\mathrm{app})}(\mu \mathrm{M})$ & $82 \pm 3$ & $2.6 \pm 0.3(7.2 \pm 0.6)^{e}$ \\
\hline $\mathrm{k}_{\text {cat (app) }} / \mathrm{K}_{\mathrm{m} \text { (app) }}\left(\min ^{-1} \mu \mathrm{M}^{-1}\right)$ & 0.10 & $11(5.1)^{e}$ \\
\hline $\mathrm{K}_{\mathrm{i}}(1$-cyclohexenoate $)(\mu \mathrm{M})$ & $182 \pm 6$ & $5.2 \pm 0.4(16)^{f}$ \\
\hline
\end{tabular}

${ }^{a}$ Data previously reported (5).

$b_{4 \mathrm{xy}}=462$ or 455 for the Trp355Phe mutant or wild type nikD, respectively.

${ }^{c}$ Ranges for spectral properties are based on data for various preparations, including those listed in Table 2 and previously reported results (5).

${ }^{d}$ Average of values obtained for six preparations.

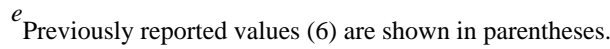

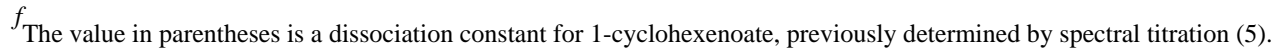

